Appl. Set-Valued Anal. Optim. 1 (2019), No. 2, pp. 187-201

Available online at http://asvao.biemdas.com

https://doi.org/10.23952/asvao.1.2019.2.07

\title{
SOME HOMOGENEOUS $q$-DIFFERENCE OPERATORS AND THE ASSOCIATED GENERALIZED HAHN POLYNOMIALS
}

\author{
HARI M. SRIVASTAVA ${ }^{1, *}$, SAMA ARJIKA ${ }^{2}$, ABEY SHERIF KELIL $^{3}$ \\ ${ }^{1}$ Department of Mathematics and Statistics, University of Victoria, Victoria, British Columbia V8W3R4, Canada \\ ${ }^{2}$ Department of Mathematics and Informatics, Faculty of Sciences and Technics, \\ University of Agadez, Post Box 199, Agadez, Niger \\ ${ }^{3}$ Department of Mathematics and Applied Mathematics, University of Pretoria, \\ Private Bag X20, Hatfield, 0028 Pretoria, Gauteng, South Africa
}

\begin{abstract}
In this paper, we first construct the homogeneous $q$-shift operator $\widetilde{E}\left(a, b ; D_{q}\right)$ and the homogeneous $q$-difference operator $\widetilde{L}\left(a, b ; \Theta_{x, y}\right)$. We then apply these operators in order to represent and investigate a family of generalized Cauchy polynomials and a general form of the $q$-Hahn polynomials. We derive some $q$-identities such as generating functions, extended generating functions, Mehler's formula and Rogers' formula for these $q$-polynomials. Relevant connections of the $q$-identities presented here with a number of known or new results associated with various specialized families of $q$-polynomials are also considered.
\end{abstract}

Keywords. Cauchy polynomials; Hahn polynomials; Rogers' formula; Cauchy polynomials; Generating functions.

2010 Mathematics Subject Classification. 05A30, 33D15, 33D45.

\section{INTRODUCTION}

We adopt the commonly used conventions and notations for basic (or $q$-) series and basic (or $q$-) polynomials. For the convenience of the reader, we provide a summary of the mathematical notations and definitions to be used in this paper. We refer the reader to the general references (see, for example, $[7,11,16,18])$ for the definitions and notations.

Throughout this paper, we assume $0<q<1$. For a complex numbers $a$, the $q$-shifted factorials are defined by

$$
(a ; q)_{0}:=1 \quad \text { and } \quad(a ; q)_{n}:=\prod_{k=0}^{n-1}\left(1-a q^{k}\right) \quad(n \in \mathbb{N}:=\{1,2,3, \cdots\})
$$

and, for large $n$, we have

$$
(a ; q)_{\infty}:=\prod_{k=0}^{\infty}\left(1-a q^{k}\right)
$$

\footnotetext{
${ }^{*}$ Corresponding author.

E-mail addresses: harimsri@math.uvic.ca (H.M. Srivastava), rjksama2008@gmail.com (S. Arjika), abeysh2001@gmail. com (A.S. Kelin).
}

Received April 7, 2019; Accepted June 23, 2019.

(C)2019 Applied Set-Valued Analysis and Optimization 
The $q$-numbers and the $q$-factorials are defined as follows:

$$
[n]_{q}:=\frac{1-q^{n}}{1-q}, \quad[n]_{q} !:=\prod_{k=1}^{n}\left(\frac{1-q^{k}}{1-q}\right) \quad \text { and } \quad[0]_{q} !:=1 .
$$

The $q$-binomial coefficients are given by

$$
\left[\begin{array}{l}
n \\
k
\end{array}\right]_{q}:=\frac{[n]_{q} !}{[k]_{q} ![n-k]_{q} !}=\left[\begin{array}{c}
n \\
n-k
\end{array}\right]_{q}
$$

The basic (or $q^{-}$) hypergeometric function of the variable $z$ and with $\mathfrak{r}$ numerator and $\mathfrak{s}$ denominator parameters (see, for details, the monographs by Slater [16, Chapter 3] and by Srivastava and Karlsson [18, p. 347, Eq. (272)]; see also [11]) is defined as follows:

$$
{ }_{\mathfrak{r}} \Phi_{\mathfrak{s}}\left[\begin{array}{c}
a_{1}, a_{2}, \cdots, a_{\mathfrak{r}} ; \\
b_{1}, b_{2}, \cdots, b_{\mathfrak{s}} ;
\end{array}\right]=\sum_{n=0}^{\infty}\left[(-1)^{n} q^{\left.\left(\begin{array}{c}
n \\
2
\end{array}\right)\right]^{1+s-r}} \frac{\left(a_{1}, a_{2}, \cdots, a_{\mathfrak{r}} ; q\right)_{n}}{\left(b_{1}, b_{2}, \cdots, b_{\mathfrak{s}} ; q\right)_{n}} \frac{z^{n}}{(q ; q)_{n}} .\right.
$$

Basic or $q$-hypergeometric series and various associated families of $q$-polynomials are useful in a wide variety of fields including, for example, the theory of partitions, number theory, combinatorial analysis, finite vector spaces, Lie theory, particle physics, non-linear electric circuit theory, mechanical engineering, theory of heat conduction, quantum mechanics, cosmology, and statistics (see [18, pp. 346$351]$ and the references cited therein).

Here, in our present investigation, we are mainly concerned with the Cauchy polynomials $p_{n}(x, y)$ as given below (see [7]):

$$
p_{n}(x, y):=(x-y)(x-q y) \cdots\left(x-q^{n-1} y\right)=(y / x ; q)_{n} x^{n}
$$

with the following generating function ([5]):

$$
\sum_{k=0}^{\infty} p_{n}(x, y) \frac{t^{n}}{(q ; q)_{k}}=\frac{(y t ; q)_{\infty}}{(x t ; q)_{\infty}}
$$

where [5]

$$
p_{n}(x, y)=(-1)^{n} q^{\left(\begin{array}{c}
n \\
2
\end{array}\right)} p_{n}\left(y, q^{1-n} x\right)
$$

and

$$
p_{n-k}\left(x, q^{1-n} y\right)=(-1)^{n-k} q^{\left(\begin{array}{l}
k \\
2
\end{array}\right)-\left(\begin{array}{l}
n \\
2
\end{array}\right)} p_{n-k}\left(y, q^{k} x\right) .
$$

The Cauchy polynomials $p_{n}(x, y)$ arise naturally in the $q$-umbral calculus (see, for details, the works by Andrews [2], Araci et al. [3], Goldman and Rota [8], Ihrig and Ismail [9], Johnson [10] and Roman [12]). The generating function (1.7) is also the homogeneous version of the Cauchy identity or the following $q$-binomial theorem (see [7]):

$$
\sum_{k=0}^{\infty} \frac{(a ; q)_{k}}{(q ; q)_{k}} z^{k}={ }_{1} \Phi_{0}\left[\begin{array}{c}
a ; \\
-;
\end{array}\right]=\frac{(a z ; q)_{\infty}}{(z ; q)_{\infty}} .
$$

Putting $a=0$, the $q$-binomial theorem (1.10) becomes Euler's identity given by (see [7])

$$
\sum_{k=0}^{\infty} \frac{z^{k}}{(q ; q)_{k}}=\frac{1}{(z ; q)_{\infty}}
$$


and its inverse relation (see [7]):

$$
\sum_{k=0}^{\infty} \frac{(-1)^{k} q^{\left(\begin{array}{c}
k \\
2
\end{array}\right)} z^{k}}{(q ; q)_{k}}=(z ; q)_{\infty}
$$

Recently, Saad and Sukhi [15] introduced the following $q$-exponential operator $R\left(b D_{q}\right)$ :

$$
R\left(b D_{q}\right)=\sum_{k=0}^{\infty} \frac{(-1)^{k} q^{\left(\begin{array}{l}
k \\
2
\end{array}\right)}}{(q ; q)_{k}}\left(b D_{q}\right)^{k}
$$

where the $q$-difference operator or the $q$-derivative, acting upon the variable a, is defined by (see [6] and [14])

Evidently, we have

$$
D_{q}\{f(a)\}=\frac{f(a)-f(q a)}{a} .
$$

$$
\lim _{q \rightarrow 1-}\left\{\frac{D_{q}\{f(a)\}}{1-q}\right\}=f^{\prime}(a),
$$

provided that the derivative $f^{\prime}(a)$ exists. Moreover, it is easily seen that

$$
R\left(y D_{q}\right)\left\{x^{n}\right\}=p_{n}(x, y) .
$$

Suppose that the operator $R\left(y D_{q}\right)$ acts upon the variable $x$. We then have the following consequence (see [15] and [17]):

$$
\begin{gathered}
R\left(y D_{q}\right)\left\{\frac{1}{(x t ; q)_{\infty}}\right\}=\frac{(y t ; q)_{\infty}}{(x t ; q)_{\infty}} \\
R\left(y D_{q}\right)\left\{\frac{1}{(x t, x s ; q)_{\infty}}\right\}=\frac{(y s ; q)_{\infty}}{(x t, x s ; q)_{\infty}}{ }_{1} \Phi_{1}\left[\begin{array}{cc}
x s ; & q ; y t \\
y s ;
\end{array}\right], \\
R\left(y D_{q}\right)\left\{\frac{(x v ; q)_{\infty}}{(x t, x s ; q)_{\infty}}\right\}=\frac{(y s ; q)_{\infty}}{(x s ; q)_{\infty}}{ }_{2} \Phi_{1}\left[\begin{array}{cc}
v / t, y / x ; & q ; x t \\
y s ;
\end{array}\right] .
\end{gathered}
$$

Srivastava and Abdlhusein [17] showed by setting $v=0$ in (1.18), that

$$
R\left(y D_{q}\right)\left\{\frac{1}{(x t, x s ; q)_{\infty}}\right\}=\frac{(y s ; q)_{\infty}}{(x s ; q)_{\infty}} 2 \Phi_{1}\left[\begin{array}{c}
y / x, 0 ; \\
y s ;
\end{array}\right] .
$$

Comparing (1.19) with (1.17), we get the following $q$-hypergeometric transformation:

$$
{ }_{2} \Phi_{1}\left[\begin{array}{cc}
y / x, 0 ; & \\
y s ; & q ; x t
\end{array}\right]=\frac{1}{(x t ; q)_{\infty}}{ }_{1} \Phi_{1}\left[\begin{array}{c}
x s ; \\
y s ;
\end{array}\right] .
$$

Motivated by the recent investigations by (for example) [4], Saad and Sukhi [15] and Srivastava and Abdlhusein [17], we aim here to introduce the (presumably new) homogeneous $q$-difference operators $\widetilde{E}\left(a, b ; D_{q}\right)$ and $\widetilde{T}\left(a, b ; \Theta_{x, y}\right)$.

Definition 1.1. The first homogeneous $q$-difference operator $\widetilde{E}\left(a, b ; D_{q}\right)$ is defined by

$$
\widetilde{E}\left(a, b ; D_{q}\right)=\sum_{k=0}^{\infty} \frac{(-1)^{k} q^{\left(\begin{array}{l}
k \\
2
\end{array}\right)}(a ; q)_{k}}{(q ; q)_{k}}\left(b D_{q}\right)^{k} .
$$


Remark 1.1. When compared with $R\left(y D_{q}\right)$, the homogeneous $q$-difference operator $\widetilde{E}\left(a, b ; D_{q}\right)$ given by (1.21) involves two parameters. Clearly, the operator $R\left(y D_{q}\right)$ can be considered as a special case of this operator in (1.21) for $a=0$, that is,

$$
\widetilde{E}\left(0, b ; D_{q}\right)=R\left(b D_{q}\right) .
$$

Definition 1.2. The second homogeneous $q$-difference operator $\widetilde{L}\left(a, b ; \Theta_{x, y}\right)$ is defined by

$$
\widetilde{L}\left(a, b ; \Theta_{x, y}\right)=\sum_{k=0}^{\infty} \frac{q^{\left(\begin{array}{l}
k \\
2
\end{array}\right)}(a ; q)_{k}}{(q ; q)_{k}}\left(b \Theta_{x, y}\right)^{k},
$$

where (see [6] and [14])

$$
\Theta_{x, y}\{f(x, y)\}=\frac{f\left(q^{-1} x, y\right)-f(x, q y)}{q^{-1} x-y},
$$

which acts upon functions of suitable variables $x$ and $y$ (see [17] and [14]), and

$$
\Theta_{x, y}^{k}\left\{p_{n}(y, x)\right\}=(-1)^{k} \frac{(q ; q)_{n}}{(q ; q)_{n-k}} p_{n-k}(y, x) \quad \text { and } \quad \Theta_{x, y}^{k}\left\{\frac{(x t ; q)_{\infty}}{(y t ; q)_{\infty}}\right\}=(-t)^{k} \frac{(x t ; q)_{\infty}}{(y t ; q)_{\infty}} .
$$

Remark 1.2. The homogeneous $q$-difference operator $\widetilde{L}\left(a, b ; \Theta_{x, y}\right)$ given by (1.23) involves two parameters. It can be considered as a generalization of the homogeneous $q$-difference operator $L\left(b \Theta_{x, y}\right)$, which was introduced by Saad and Sukhi [14]. In its special case when $a=0$, we have

$$
\widetilde{L}\left(0, b ; \Theta_{x, y}\right)=L\left(b \Theta_{x, y}\right) .
$$

The definition (1.23) is motivated by the natural question of extending such generating functions as (for example) Mehler's formula and Rogers-type formula for a general form for the Hahn polynomials $h_{n}(x, y, a, b \mid q)$. The operators defined here (1.21) and (1.23) turn out to be suitable for dealing with the generalized Cauchy polynomials $p_{n}(x, y, a)$ as well as the generalized Hahn polynomials $h_{n}(x, y, a, b \mid q)$. They are then applied in order to represent and investigate such $q$-identities as generating functions, extended generating functions, Mehler's formula and Rogers-type formula for the polynomials $p_{n}(x, y, a)$ and $h_{n}(x, y, a, b \mid q)$.

\section{Generalized Cauchy polynomials $p_{n}(x, y, a)$}

In this section, we introduce the generalized Cauchy polynomials $p_{n}(x, y, a)$. We then represent these polynomials $p_{n}(x, y, a)$ by means of the homogeneous $q$-difference operator $\widetilde{E}\left(a, b ; D_{q}\right)$ and derive their generating function. We also use the operational formula for $p_{n}(x, y, a)$ in order to establish an extended generating function, Mehler's formula and the Rogers formula for the generalized Cauchy polynomials $p_{n}(x, y, a)$.

Let us start by recalling following Leibniz rule (see [13]):

$$
D_{q}^{n}\{f(x) g(x)\}=\sum_{k=0}^{n}\left[\begin{array}{l}
n \\
k
\end{array}\right]_{q} q^{k(k-n)} D_{q}^{k}\{f(x)\} D_{q}^{n-k}\left\{g\left(q^{k} x\right)\right\} .
$$

For $f(x)=x^{k}, f(x)=(x t ; q)_{\infty}$ and

$$
f(x)={ }_{1} \Phi_{1}\left[\begin{array}{cc}
a ; & \\
& q ; b x \\
0 ; &
\end{array}\right]
$$


we have the following identities:

$$
D_{q}^{n}\left\{x^{k}\right\}=\left(q^{k-n+1} ; q\right)_{n} x^{k-n}, \quad D_{q}^{n}\left\{(x t ; q)_{\infty}\right\}=q^{\left(\begin{array}{c}
n \\
2
\end{array}\right)} t^{n} \frac{(x t ; q)_{\infty}}{(x t ; q)_{n}}
$$

and

$$
D_{q}^{n}\left\{{ }_{1} \Phi_{1}\left[\begin{array}{cc}
a ; & \\
& q ; b x \\
0 ; &
\end{array}\right]\right\}=(-1)^{n} b^{n}(a ; q){ }_{n} q^{\left(\begin{array}{l}
n \\
2
\end{array}\right)}{ }_{1} \Phi_{1}\left[\begin{array}{cc}
a q^{n} ; & \\
0 ; & \left.q ; b q^{n}\right] .
\end{array}\right]
$$

Suppose now that the operator $D_{q}$ acts upon the variable $s$. If we set $f(s)=s^{n}$ and $g(s)=s^{m}$ or, alternatively,

and

$$
f(s)={ }_{1} \Phi_{1}\left[\begin{array}{cc}
a ; & \\
& q ; b s q^{k} \\
0 ; &
\end{array}\right]
$$

$$
g(s)=\frac{1}{\left(x s q^{k} ; q\right)_{\infty}},
$$

and make use of (2.2) and (2.1), we get the following identities to be used in our investigation:

$$
\left(q^{n+m-k+1} ; q\right)_{k}=\sum_{j=0}^{k}\left[\begin{array}{l}
k \\
j
\end{array}\right]_{q} q^{j(j-k+m)}\left(q^{n-j+1} ; q\right)_{j}\left(q^{m-k+j+1} ; q\right)_{k-j}
$$

and

$$
\begin{aligned}
& D_{q}^{n}\left\{\frac{{ }_{1} \Phi_{1}\left[\begin{array}{cr}
a ; \\
& q ; b s q^{k}
\end{array}\right]}{\left(x s q^{k} ; q\right)_{\infty}}\right\} \\
& =\frac{1}{(x s ; q)_{\infty}} \sum_{j=0}^{n}\left[\begin{array}{c}
n \\
j
\end{array}\right]_{q} q^{j(j-n+k)+\left(\begin{array}{l}
j \\
2
\end{array}\right)} x^{j}(x s ; q)_{j} D_{q}^{n-j}\left\{{ }_{1} \Phi_{1}\left[\begin{array}{cc}
a ; & \\
0 ; b s q^{j+k} &
\end{array}\right]\right\} .
\end{aligned}
$$

Next, recalling that the homogeneous $q$-difference operator $\widetilde{E}\left(a, b ; D_{q}\right)$ is defined by (1.21) (see Definition 1.1) as well as its special case when $a=0$ by Remark 1.1, we are led to Proposition 2.1 below.

Proposition 2.1. Suppose that the operator $D_{q}$ acts upon the variable $x$. Then

$$
\begin{gathered}
\widetilde{E}\left(a, y ; D_{q}\right)\left\{\frac{1}{(x t ; q)_{\infty}}\right\}=\frac{1}{(x t ; q)_{\infty}}{ }_{1} \Phi_{1}\left[\begin{array}{c}
a ; \\
\\
0 ;
\end{array}\right], \\
\widetilde{E}\left(a, y ; D_{q}\right)\left\{\frac{1}{(x t, x s ; q)_{\infty}}\right\} \\
=\frac{1}{(x t, x s ; q)_{\infty}} \sum_{n=0}^{\infty} \frac{(-1)^{n} q^{\left(\begin{array}{l}
n \\
2
\end{array}\right)(a ; q)_{n}(s y)^{n}}}{(q ; q)_{n}}{ }_{2} \Phi_{1}\left[\begin{array}{c}
a q^{n}, x s ; \\
0 ; q^{n} y t
\end{array}\right]
\end{gathered}
$$


and

$$
\begin{aligned}
& \widetilde{E}\left(a, y ; D_{q}\right)\left\{\frac{x^{k}}{(x t ; q)_{\infty}}\right\} \\
& \quad=\frac{x^{k}}{(x t ; q)_{\infty}} \sum_{n=0}^{\infty} \frac{(-1)^{n} q^{\left(\begin{array}{c}
n \\
2
\end{array}\right)(a ; q)_{n}(y t)^{n}}}{(q ; q)_{n}}{ }_{3} \Phi_{2}\left[\begin{array}{c}
q^{-k}, a q^{n}, x t ; \\
0,0 ;
\end{array} ; \frac{y}{x} q^{n+k}\right] .
\end{aligned}
$$

Remark 2.1. Upon setting $k=0$ in the assertion (2.8), if we make use of the following identity:

$$
{ }_{3} \Phi_{2}\left[\begin{array}{c}
1, a q^{n}, x t ; \\
0,0 ;
\end{array} \quad q ; \frac{y}{x} q^{n}\right]=1,
$$

we get (2.6).

Theorem 2.1. Suppose that the operator $D_{q}$ acts upon the variable s. Then

$$
\begin{aligned}
\widetilde{E}\left(a, t ; D_{q}\right) & \left\{\frac{(y s ; q)_{\infty}}{(x s ; q)_{\infty}}{ }_{1} \Phi_{1}\left[\begin{array}{c}
a ; \\
0 ;
\end{array}\right]\right\} \\
= & \frac{(y s ; q)_{\infty}}{(x s ; q)_{\infty}} \sum_{n=0}^{\infty} \sum_{k=0}^{\infty} \sum_{j=0}^{\infty} \frac{(-1)^{j+k} q^{\left(\begin{array}{l}
k \\
2
\end{array}\right)+\left(\begin{array}{l}
n \\
2
\end{array}\right)+\left(\begin{array}{c}
n+j+k \\
2
\end{array}\right)}}{(q ; q)_{k}(q ; q)_{n}(q ; q)_{j}} \\
& \cdot \frac{(a ; q)_{n}(a ; q)_{n+j+k}(x s ; q)_{k}}{(y s ; q)_{k}}{ }_{1} \Phi_{1}\left[\begin{array}{c}
a q^{n} ; \\
0 ; b s q^{j+k+n}
\end{array}\right] t^{n+j+k} .
\end{aligned}
$$

Proof. We observe that

$$
\begin{aligned}
\widetilde{E}\left(a, t ; D_{q}\right) & \left\{\frac{(y s ; q)_{\infty}}{}(x s ; q)_{\infty}{ }_{1} \Phi_{1}\left[\begin{array}{cc}
a ; & \\
0 ; & q ; b s]\}
\end{array}\right]\right. \\
& =\sum_{n=0}^{\infty} \frac{(-1)^{n} q^{\left(\begin{array}{c}
n \\
2
\end{array}\right)(a ; q)_{n} t^{n}}}{(q ; q)_{n}} D_{q}^{n}\left\{\frac{(y s ; q)_{\infty}}{(x s ; q)_{\infty}}{ }_{1} \Phi_{1}\left[\begin{array}{cc}
a ; & q ; b s]\} \\
0 ;
\end{array}\right]\right\}
\end{aligned}
$$

By applying (2.1), the right-hand side of (2.10) becomes

$$
\sum_{n=0}^{\infty} \frac{(-1)^{n} q^{\left(\begin{array}{l}
n \\
2
\end{array}\right)(a ; q)_{n} t^{n}}}{(q ; q)_{n}} \sum_{k=0}^{n}\left[\begin{array}{l}
n \\
k
\end{array}\right]_{q} q^{k(k-n)} D_{q}^{k}\left\{(y s ; q)_{\infty}\right\} D_{q}^{n-k}\left\{\frac{{ }_{1} \Phi_{1}\left[\begin{array}{c}
a ; \\
q ; b s q^{k} \\
0 ;
\end{array}\right]}{\left(x s q^{k} ; q\right)_{\infty}}\right\}
$$


or, equivalently,

$$
\sum_{n=0}^{\infty} \sum_{k=n}^{\infty} \frac{(-1)^{n} q^{\left(\begin{array}{c}
n \\
2
\end{array}\right)+k(k-n)+\left(\begin{array}{c}
k \\
2
\end{array}\right) t^{n} y^{k}(a ; q)_{n}}\left(y s q^{k} ; q\right)_{\infty} D_{q}^{n-k}}{(q ; q)_{k}(q ; q)_{n-k}}\left\{\frac{{ }_{1} \Phi_{1}\left[\begin{array}{cc}
a ; & \\
& q ; b s q^{k} \\
0 ;
\end{array}\right]}{\left(x s q^{k} ; q\right)_{\infty}}\right\}
$$

which, upon replacing $n$ by $n+k$, yields

$$
(y s ; q)_{\infty} \sum_{n=0}^{\infty} \sum_{k=0}^{\infty} \frac{(-1)^{n+k} q^{\left(\begin{array}{c}
n+k \\
2
\end{array}\right)-k n+\left(\begin{array}{c}
k \\
2
\end{array}\right) t^{n+k} y^{k}(a ; q)_{n+k}}}{(y s ; q)_{k}(q ; q)_{k}(q ; q)_{n}} D_{q}^{n}\left\{\frac{{ }_{1} \Phi_{1}\left[\begin{array}{c}
a ; \\
q ; b s q^{k} \\
0 ;
\end{array}\right]}{\left(x s q^{k} ; q\right)_{\infty}}\right\} .
$$

Now, by using (2.5) in (2.13), we find that

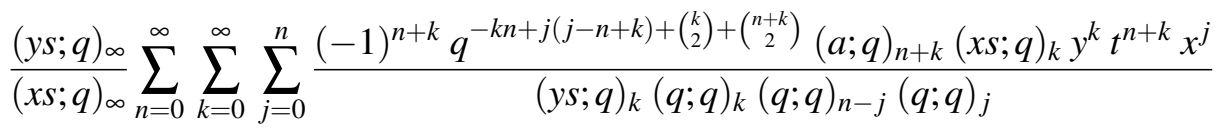

$$
\begin{aligned}
& \cdot D_{q}^{n-j}\left\{{ }_{1} \Phi_{1}\left[\begin{array}{cc}
a ; & \\
& q ; b s q^{j+k} \\
0 ; &
\end{array}\right]\right\} \\
& =\frac{(y s ; q)_{\infty}}{(x s ; q)_{\infty}} \sum_{n=0}^{\infty} \sum_{k=0}^{\infty} \sum_{j=0}^{\infty} \frac{(-1)^{n+j+k} q^{-k n-j n+\left(\begin{array}{c}
k \\
2
\end{array}\right)+\left(\begin{array}{c}
n+j+k \\
2
\end{array}\right)(a ; q)_{n+j+k}(x s ; q)_{k} y^{k} t^{n+j+k} x^{j}}}{(y s ; q)_{k}(q ; q)_{k}(q ; q)_{n}(q ; q)_{j}} \\
& \cdot D_{q}^{n}\left\{{ }_{1} \Phi_{1}\left[\begin{array}{cc}
a ; & \\
0 ; & q s q^{j+k} \\
0 ; &
\end{array}\right]\right\}
\end{aligned}
$$

in which the right-hand side can be simplified to the following form:

$$
\begin{aligned}
& \frac{(y s ; q)_{\infty}}{(x s ; q)_{\infty}} \sum_{n=0}^{\infty} \sum_{k=0}^{\infty} \sum_{j=0}^{\infty} \frac{(-1)^{j+k} q^{\left(\begin{array}{c}
k \\
2
\end{array}\right)+\left(\begin{array}{c}
n \\
2
\end{array}\right)+\left(\begin{array}{c}
n+j+k \\
2
\end{array}\right)}(a ; q)_{n}(a ; q)_{n+j+k}(x s ; q)_{k} b^{n} y^{k} t^{n+j+k} x^{j}}{(y s ; q)_{k}(q ; q)_{k}(q ; q)_{n}(q ; q)_{j}} \\
& \cdot{ }_{1} \Phi_{1}\left[\begin{array}{cc}
a q^{n} ; & \\
0 ; & q s q^{j+k+n}
\end{array}\right] .
\end{aligned}
$$

By appropriately combining the above observations, we get the assertion (2.9) of Theorem 2.1.

Definition 2.1. In terms of the $q$-shifted factorial, the generalized Cauchy polynomials $p_{n}(x, y, a)$ are defined by

with

$$
p_{n}(x, y, a)=\sum_{k=0}^{n}\left[\begin{array}{l}
n \\
k
\end{array}\right]_{q}(-1)^{k} q^{\left(\begin{array}{l}
k \\
2
\end{array}\right)}(a ; q)_{k} x^{n-k} y^{k}
$$

$$
p_{n}(0, y, a)=(-1)^{n} q^{\left(\begin{array}{c}
n \\
2
\end{array}\right)}(a ; q)_{n} y^{n}, \quad p_{n}(x, 0, a)=x^{n}
$$


and

$$
p_{n}(x, y, 0)=\sum_{k=0}^{n}\left[\begin{array}{l}
n \\
k
\end{array}\right]_{q}(-1)^{k} q^{\left(\begin{array}{c}
k \\
2
\end{array}\right)} x^{n-k} y^{k}
$$

Proposition 2.2. The following operational formula holds true:

$$
\widetilde{E}\left(a, y ; D_{q}\right)\left\{x^{n}\right\}=p_{n}(x, y, a) .
$$

Proof. In order to demonstrate the assertion (2.19) of Proposition 2.2, we see that

$$
\begin{aligned}
\widetilde{E}\left(a, y ; D_{q}\right)\left\{x^{n}\right\} & =\sum_{k=0}^{\infty} \frac{(-1)^{k} q^{\left(\begin{array}{l}
k \\
2
\end{array}\right)(a ; q)_{k}}}{(q ; q)_{k}}\left(y D_{q}\right)^{k}\left\{x^{n}\right\} \\
& =\sum_{k=0}^{\infty} \frac{(-1)^{k} q^{\left(\begin{array}{l}
k \\
2
\end{array}\right)(a ; q)_{k}}}{(q ; q)_{k}} \frac{(q ; q)_{n}}{(q ; q)_{n-k}} y^{k} x^{n-k}=p_{n}(x, y, a) .
\end{aligned}
$$

Theorem 2.2. [Generating function for $p_{n}(x, y, a)$ ] The following generating function holds true:

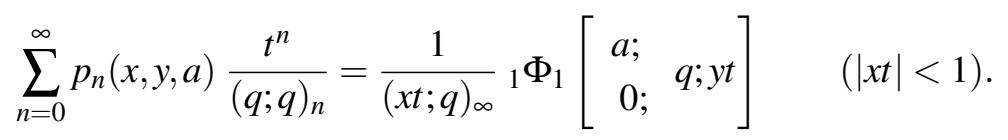

Proof. Let us suppose that the operator $D_{q}$ acts upon the variable $x$. We then find that

$$
\begin{aligned}
\sum_{n=0}^{\infty} p_{n}(x, y, a) \frac{t^{n}}{(q ; q)_{n}} & =\sum_{n=0}^{\infty} \widetilde{E}\left(a, y ; D_{q}\right)\left\{x^{n}\right\} \frac{t^{n}}{(q ; q)_{n}} \\
& =\widetilde{E}\left(a, y ; D_{q}\right)\left\{\sum_{n=0}^{\infty} \frac{(x t)^{n}}{(q ; q)_{n}}\right\} \\
& =\widetilde{E}\left(a, y ; D_{q}\right)\left\{\frac{1}{(x t ; q)_{\infty}}\right\} \\
& =\frac{1}{(x t ; q)_{\infty}}{ }_{1} \Phi_{1}\left[\begin{array}{c}
a ; \\
q ; y t \\
0 ;
\end{array}\right] \quad(|x t|<1),
\end{aligned}
$$

which evidently completes the proof of the assertion (2.21) of Theorem 2.2.

Theorem 2.3. [Extended generating function for $\left.p_{n}(x, y, a)\right]$ It is asserted that

$$
\begin{aligned}
& \sum_{n=0}^{\infty} p_{n+k}(x, y, a) \frac{t^{n}}{(q ; q)_{n}} \\
& \quad=\frac{x^{k}}{(x t ; q)_{\infty}} \sum_{n=0}^{\infty} \frac{(-1)^{n} q^{\left(\begin{array}{c}
n \\
2
\end{array}\right)(a ; q)_{n}(y t)^{n}}}{(q ; q)_{n}}{ }_{3} \Phi_{2}\left[\begin{array}{c}
q^{-k}, a q^{n}, x t ; \\
0,0 ;
\end{array} ; \frac{y}{x} q^{n+k}\right] \quad(|x t|<1) .
\end{aligned}
$$


Proof. We suppose that the operator $D_{q}$ acts upon the variable $s$. We then obtain

$$
\begin{aligned}
& \sum_{n=0}^{\infty} p_{n+k}(x, y, a) \frac{t^{n}}{(q ; q)_{n}} \\
& =\sum_{n=0}^{\infty} \widetilde{E}\left(a, y ; D_{q}\right)\left\{x^{n+k}\right\} \frac{t^{n}}{(q ; q)_{n}} \\
& =\widetilde{E}\left(a, y ; D_{q}\right)\left\{x^{k} \sum_{n=0}^{\infty} \frac{(x t)^{n}}{(q ; q)_{n}}\right\} \\
& =\widetilde{E}\left(a, y ; D_{q}\right)\left\{\frac{x^{k}}{(x t ; q)_{\infty}}\right\} .
\end{aligned}
$$

The proof of the assertion (2.23) of Theorem 2.3 is thus completed by applying the relation (2.8).

Remark 2.2. Upon setting $k=0$ in Theorem 2.3, we get the generating function (2.21).

Theorem 2.4. [Rogers-type formula for $p_{n}(x, y, a)$ ] The following Rogers-type formula holds true:

$$
\begin{gathered}
\sum_{n=0}^{\infty} \sum_{m=0}^{\infty} p_{n+m}(x, y, a) \frac{t^{n}}{(q ; q)_{n}} \frac{s^{m}}{(q ; q)_{m}} \\
=\frac{1}{(x t, x s ; q)_{\infty}} \sum_{n=0}^{\infty} \frac{(-1)^{n} q^{\left(\begin{array}{c}
n \\
2
\end{array}\right)(a ; q)_{n}(s y)^{n}}}{(q ; q)_{n}}{ }_{2} \Phi_{1}\left[\begin{array}{c}
a q^{n}, x s ; \\
0 ; y t q^{n} \\
(\max \{|x t|,|x s|\}<1) .
\end{array}\right.
\end{gathered}
$$

Proof. We suppose that the operator $D_{q}$ acts upon the variable $x$. We then find that

$$
\begin{aligned}
& \sum_{n=0}^{\infty} \sum_{m=0}^{\infty} p_{n+m}(x, y, a) \frac{t^{n}}{(q ; q)_{n}} \frac{s^{m}}{(q ; q)_{m}} \\
& \quad=\sum_{n=0}^{\infty} \sum_{m=0}^{\infty} \widetilde{E}\left(a, y ; D_{q}\right)\left\{x^{n+m}\right\} \frac{t^{n}}{(q ; q)_{n}} \frac{s^{m}}{(q ; q)_{m}} \\
& =\widetilde{E}\left(a, y ; D_{q}\right)\left\{\sum_{n=0}^{\infty} \frac{(x t)^{n}}{(q ; q)_{n}} \sum_{n=0}^{\infty} \frac{(x s)^{m}}{(q ; q)_{m}}\right\} \\
& =\widetilde{E}\left(a, y ; D_{q}\right)\left\{\frac{1}{(x t, x s ; q)_{\infty}}\right\} \quad(\max \{|x t|,|x s|\}<1) .
\end{aligned}
$$

The proof of Theorem 2.4 is now completed by making use of the relation (2.7).

We aim now to present an operator approach to Mehler's formula for the generalized Cauchy polynomials $p_{n}(x, y, a)$.

Theorem 2.5. [Mehler's formula for $\left.p_{n}(x, y, a)\right]$ The following Mehler-type bilinear generating function holds true for the generalized Cauchy polynomials $p_{n}(x, y, a)$ :

$$
\begin{aligned}
& \sum_{n=0}^{\infty} p_{n}(x, y, a) p_{n}(u, v, \alpha) \frac{t^{n}}{(q ; q)_{n}} \\
& \quad=\widetilde{E}\left(a, y ; D_{q}\right)\left\{\frac{1}{(u x t ; q)_{\infty}}{ }_{1} \Phi_{1}\left[\begin{array}{cc}
\alpha ; & \\
0 ; & q ; v x t
\end{array}\right]\right\} \quad(|u x t|<1) .
\end{aligned}
$$


Proof. We observe that

$$
\begin{aligned}
& \sum_{n=0}^{\infty} p_{n}(x, y, a) p_{n}(u, v, \alpha) \frac{t^{n}}{(q ; q)_{n}} \\
& \quad=\sum_{n=0}^{\infty} \widetilde{E}\left(a, y ; D_{q}\right)\left\{x^{n}\right\} p_{n}(u, v, \alpha) \frac{t^{n}}{(q ; q)_{n}} \\
& =\widetilde{E}\left(a, y ; D_{q}\right)\left\{\sum_{n=0}^{\infty} p_{n}(u, v, \alpha) \frac{(x t)^{n}}{(q ; q)_{n}}\right\} \\
& =\widetilde{E}\left(a, y ; D_{q}\right)\left\{\frac{1}{(u x t ; q)_{\infty}}{ }_{1} \Phi_{1}\left[\begin{array}{c}
\alpha ; \\
0 ; v x t
\end{array}\right]\right\} \quad(|u x t|<1),
\end{aligned}
$$

which evidently completes our demonstration of th the Mehler-type bilinear generating function (2.27) for the generalized Cauchy polynomials $p_{n}(x, y, a)$.

\section{The HOMOGENEOUS $q$-DIFFERENCE OPERATOR $\widetilde{L}\left(a, b ; \Theta_{x, y}\right)$ AND THE GENERALIZED HAHN POLYNOMIALS}

In this section, we first define the generalized Hahn polynomials $h_{n}(x, y, a, b \mid q)$ in terms of the homogeneous $q$-difference operator $\widetilde{L}\left(a, b ; \Theta_{x, y}\right)$ given by Definition 1.2. We then derive an extended generating function, a Mehler's formula and a Rogers-type formula for the generalized Hahn polynomials $h_{n}(x, y, a, b \mid q)$.

Remark 3.1. In terms of the $q$-hypergeometric function ${ }_{\mathfrak{r}} \Phi_{\mathfrak{s}}$ in (1.5), the homogeneous $q$-difference operator $\widetilde{L}\left(a, b ; \Theta_{x, y}\right)$ defined by (1.23) can be written as follows:

$$
\widetilde{L}\left(a, b ; \Theta_{x, y}\right)={ }_{1} \Phi_{1}\left[\begin{array}{ll}
a ; & \\
& q ;-b \Theta_{x, y} \\
0 ; & .
\end{array}\right]
$$

Theorem 3.1. The following operational formula holds true:

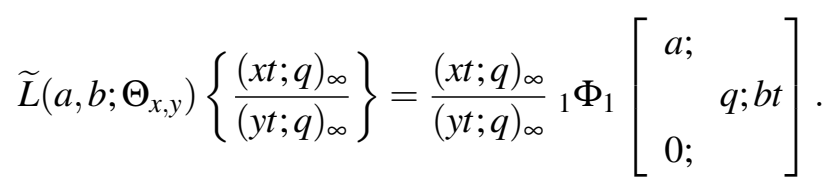

Proof. Let the function $f_{q}(x, y ; a, b ; t)$ be defined by

$$
f_{q}(x, y ; a, b ; t)=\widetilde{L}\left(a, b ; \Theta_{x, y}\right)\left\{\frac{(x t ; q)_{\infty}}{(y t ; q)_{\infty}}\right\}
$$


Then, by using (1.23) and (1.25), we have

$$
\begin{aligned}
f_{q}(x, y ; a, b ; t) & =\sum_{k=0}^{\infty} \frac{q^{\left(\begin{array}{c}
k \\
2
\end{array}\right)}(a ; q)_{k} b^{k}}{(q ; q)_{k}} \Theta_{x, y}^{k}\left\{\frac{(x t ; q)_{\infty}}{(y t ; q)_{\infty}}\right\} \\
& =\frac{(x t ; q)_{\infty}}{(y t ; q)_{\infty}} \sum_{k=0}^{\infty} \frac{(-1)^{k} q^{\left(\begin{array}{l}
k \\
2
\end{array}\right)(a ; q)_{k}}}{(q ; q)_{k}}(b t)^{k} \\
& =\frac{(x t ; q)_{\infty}}{(y t ; q)_{\infty}}{ }_{1} \Phi_{1}\left[\begin{array}{c}
a ; \\
0 ; b t \\
0 ;
\end{array}\right],
\end{aligned}
$$

which completes the proof of the assertion (3.2) of Theorem 3.1.

Definition 3.1. In terms of the $q$-shifted factorial and the Cauchy polynomials $p_{n}(x, y)$, the generalized Hahn polynomials $h_{n}(x, y, a, b \mid q)$ is defined by

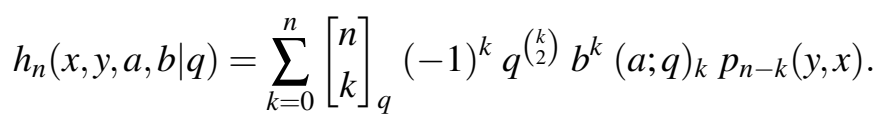

Remark 3.2. In the special case when $a=0$ and $b=z$, the $q$-polynomials $h_{n}(x, y, a, b \mid q)$ reduce to the following known trivariate $q$-polynomials $F_{n}(x, y, z ; q)$, which were investigated earlier by Mohammed (see, for details, [1]):

$$
F_{n}(x, y, z ; q):=(-1)^{n} q^{-\left(\begin{array}{l}
n \\
2
\end{array}\right)} h_{n}(x, y, 0, z \mid q)
$$

The $q$-polynomials $h_{n}(x, y, a, b \mid q)$ is a general form of the Hahn polynomials $\psi_{n}^{(a)}(x \mid q)$, for which we need to set $a=0, b=1$ and $y=a x$. Also, if we let $a=0, y=a x$ and $b=y$, we get the bivariate Hahn polynomials $\psi_{n}^{(a)}(x, y \mid q)$. We thus have

$$
h_{n}(x, a x, 0,1 \mid q)=(-1)^{n} q^{\left(\begin{array}{c}
n \\
2
\end{array}\right)} \psi_{n}^{(a)}(x \mid q) \quad \text { and } \quad h_{n}(x, a x, 0, y \mid q)=(-1)^{n} q^{\left(\begin{array}{c}
n \\
2
\end{array}\right)} \psi_{n}^{(a)}(x, y \mid q) .
$$

The polynomials in (3.5) can be represented by the homogeneous $q$-difference operator in Definition $1.2(1.23)$ as follows:

$$
h_{n}(x, y, a, b \mid q)=\widetilde{L}\left(a, b ; \Theta_{x, y}\right)\left\{p_{n}(y, x)\right\},
$$

since

$$
\begin{aligned}
\widetilde{L}\left(a, b ; \Theta_{x, y}\right)\left\{p_{n}(y, x)\right\} \\
=\sum_{k=0}^{\infty} \frac{q^{\left(\begin{array}{l}
k \\
2
\end{array}\right)}(a ; q)_{k}}{(q ; q)_{k}}\left(b \Theta_{x, y}\right)^{k}\left\{p_{n}(y, x)\right\} \\
=\sum_{k=0}^{n} \frac{(-1)^{k} q^{\left(\begin{array}{l}
k \\
2
\end{array}\right)}(a ; q)_{k} b^{k}}{(q ; q)_{k}} \frac{(q ; q)_{n}}{(q ; q)_{n-k}} p_{n-k}(y, x) \\
=h_{n}(x, y, a, b \mid q) .
\end{aligned}
$$

Theorem 3.2. [Generating function for $h_{n}(x, y, a, b \mid q)$ ] The following generating function holds true for the q-polynomials $h_{n}(x, y, a, b \mid q)$ :

$$
\sum_{n=0}^{\infty} h_{n}(x, y, a, b, \mid q) \frac{t^{n}}{(q ; q)_{n}}=\frac{(x t ; q)_{\infty}}{(y t ; q)_{\infty}}{ }_{1} \Phi_{1}\left[\begin{array}{cc}
a ; & \\
& q ; b t \\
0 ; &
\end{array}\right] \quad(\max \{|x t|,|y t|\}<1) .
$$


Proof. It is easily seen that

$$
\begin{aligned}
\sum_{n=0}^{\infty} h_{n}(x, y, a, b \mid q) \frac{t^{n}}{(q ; q)_{n}} & =\sum_{n=0}^{\infty} \widetilde{L}\left(a, b ; \Theta_{x, y}\right)\left\{p_{n}(y, x)\right\} \frac{t^{n}}{(q ; q)_{n}} \\
& =\widetilde{L}\left(a, b ; \Theta_{x, y}\right)\left\{\sum_{n=0}^{\infty} p_{n}(y, x) \frac{t^{n}}{(q ; q)_{n}}\right\} \\
& =\widetilde{L}\left(a, b ; \Theta_{x, y}\right)\left\{\frac{(x t ; q)_{\infty}}{(y t ; q)_{\infty}}\right\} \\
& =\frac{(x t ; q)_{\infty}}{(y t ; q)_{\infty}}{ }_{1} \Phi_{1}\left[\begin{array}{c}
a ; \\
0 ; b t
\end{array}\right],
\end{aligned}
$$

which evidently completes the proof of the assertion (3.10) of Theorem 3.2.

Theorem 3.3. [Extended generating function for $h_{n}(x, y, a, b \mid q)$ ] The following extended generating function holds true for the q-polynomials $h_{n}(x, y, a, b \mid q)$ :

$$
\sum_{n=0}^{\infty} h_{n+k}(x, y, a, b \mid q) \frac{t^{n}}{(q ; q)_{n}}=\widetilde{L}\left(a, b ; \Theta_{x, y}\right)\left\{\frac{(x t ; q)_{\infty}}{(x t ; q)_{k}(y t ; q)_{\infty}} p_{k}(y, x)\right\}
$$

Proof. We observe that

$$
\begin{aligned}
\sum_{n=0}^{\infty} h_{n+k}(x, y, a, b \mid q) \frac{t^{n}}{(q ; q)_{n}} & \\
= & \sum_{n=0}^{\infty} \widetilde{L}\left(a, b ; \Theta_{x, y}\right)\left\{p_{n+k}(y, x)\right\} \frac{t^{n}}{(q ; q)_{n}} \\
& =\widetilde{L}\left(a, b ; \Theta_{x, y}\right)\left\{p_{k}(y, x) \sum_{n=0}^{\infty} p_{n}\left(y, q^{k} x\right) \frac{t^{n}}{(q ; q)_{n}}\right\} \\
& =\widetilde{L}\left(a, b ; \Theta_{x, y}\right)\left\{\frac{\left(x t q^{k} ; q\right)_{\infty}}{(y t ; q)_{\infty}} p_{k}(y, x)\right\} \\
& =\widetilde{L}\left(a, b ; \Theta_{x, y}\right)\left\{\frac{(x t ; q)_{\infty}}{(x t ; q)_{k}(y t ; q)_{\infty}} p_{k}(y, x)\right\},
\end{aligned}
$$

which evidently completes the proof of the assertion (3.10) of Theorem 3.3.

Remark 3.3. Upon setting $k=0$ in (3.12), we get the generating function (3.10) for the $q$-polynomials $h_{n}(x, y, a, b \mid q)$. 
Theorem 3.4. [Rogers-type formula for $h_{n}(x, y, a, b \mid q)$ ] The following Rogers-type formula holds true for the q-polynomials $h_{n}(x, y, a, b \mid q)$ :

$$
\begin{aligned}
& \sum_{n=0}^{\infty} \sum_{m=0}^{\infty} h_{n+m}(x, y, a, b \mid q) \frac{t^{n}}{(q ; q)_{n}} \frac{s^{m}}{(q ; q)_{m}} \\
& =\widetilde{L}\left(a, b ; \Theta_{x, y}\right)\left\{\frac{(x s ; q)_{\infty}}{(y s ; q)_{\infty}}{ }_{2} \Phi_{1}\left[\begin{array}{cc}
x / y, 0 ; & \\
x s ; & q ; y t
\end{array}\right]\right\} \\
& =\widetilde{L}\left(a, b ; \Theta_{x, y}\right)\left\{\frac{(x s ; q)_{\infty}}{(y t, y s ; q)_{\infty}}{ }_{1} \Phi_{1}\left[\begin{array}{cc}
y s ; & \\
& q ; ; x t
\end{array}\right]\right\} \text {. }
\end{aligned}
$$

Proof. In order to prove the first assertion in (3.14), we have

$$
\begin{aligned}
& \sum_{n=0}^{\infty} \sum_{m=0}^{\infty} h_{n+m}(x, y, a, b \mid q) \frac{t^{n}}{(q ; q)_{n}} \frac{s^{m}}{(q ; q)_{m}} \\
& =\sum_{n=0}^{\infty} \sum_{m=0}^{\infty} \widetilde{L}\left(a, b ; \Theta_{x, y}\right)\left\{p_{n+m}(y, x)\right\} \frac{t^{n}}{(q ; q)_{n}} \frac{s^{m}}{(q ; q)_{m}} \\
& =\widetilde{L}\left(a, b ; \Theta_{x, y}\right)\left\{\sum_{n=0}^{\infty} p_{n}(y, x) \frac{t^{n}}{(q ; q)_{n}} \sum_{m=0}^{\infty} p_{m}\left(y, q^{n} x\right) \frac{s^{m}}{(q ; q)_{m}}\right\} \\
& =\widetilde{L}\left(a, b ; \Theta_{x, y}\right)\left\{\sum_{n=0}^{\infty} p_{n}(y, x) \frac{t^{n}}{(q ; q)_{n}} \frac{\left(x s q^{n} ; q\right)_{\infty}}{(y s ; q)_{\infty}}\right\} \\
& =\widetilde{L}\left(a, b ; \Theta_{x, y}\right)\left\{\frac{(x s ; q)_{\infty}}{(y s ; q)_{\infty}} \sum_{n=0}^{\infty} p_{n}(y, x) \frac{t^{n}}{(x s ; q)_{n}(q ; q)_{n}}\right\} \\
& =\widetilde{L}\left(a, b ; \Theta_{x, y}\right)\left\{\frac{(x s ; q)_{\infty}}{(y s ; q)_{\infty}} \sum_{n=0}^{\infty} \frac{(x / y ; q)_{n}(y t)^{n}}{(x s ; q)_{n}(q ; q)_{n}}\right\}
\end{aligned}
$$

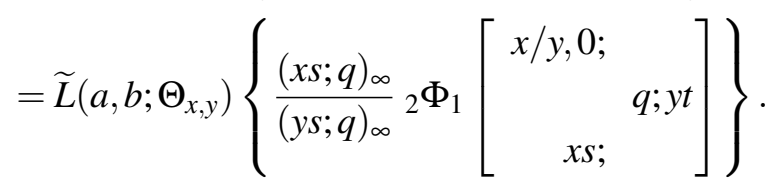

The proof of the second assertion in (3.14) of Theorem 3.4 would follow when we replace $y$ by $x$ and $x$ by $y$ in (1.20).

Finally, we prove a Mehler-type bilinear generating function for the generalized Hahn polynomias $h_{n}(x, y, a, b \mid q)$.

Theorem 3.5. [Mehler's formula for $\left.h_{n}(x, y, a, b \mid q)\right]$ The following Mehler-type bilinear generating function holds true for $h_{n}(x, y, a, b \mid q)$ :

$$
\begin{aligned}
\sum_{n=0}^{\infty} h_{n}(x, y, a, b \mid q) h_{n}(u, v, c, d \mid q) \frac{t^{n}}{(q ; q)_{n}} & \\
& =\widetilde{L}\left(a, b ; \Theta_{x, y}\right)\left\{\frac{(x t ; q)_{\infty}}{(y t ; q)_{\infty}}{ }_{3} \Phi_{3}\left[\begin{array}{rr}
x / y, u / v, c ; & \\
0,0, x t ; &
\end{array}\right]\right\} .
\end{aligned}
$$


Proof. It is observed that

$$
\begin{aligned}
& \sum_{n=0}^{\infty} h_{n}(x, y, a, b \mid q) h_{n}(u, v, c, d \mid q) \frac{t^{n}}{(q ; q)_{n}} \\
& =\sum_{n=0}^{\infty} \widetilde{L}\left(a, b ; \Theta_{x, y}\right)\left\{p_{n}(y, x)\right\} h_{n}(u, v, c, d \mid q) \frac{t^{n}}{(q ; q)_{n}} \\
& =\widetilde{L}\left(a, b ; \Theta_{x, y}\right)\left\{\sum_{n=0}^{\infty} p_{n}(y, x) \frac{t^{n}}{(q ; q)_{n}} \sum_{k=0}^{n}\left[\begin{array}{l}
n \\
k
\end{array}\right]_{q}(-1)^{k} q^{\left(\begin{array}{l}
k \\
2
\end{array}\right) d^{k}(c ; q)_{k} p_{n-k}(v, u)}\right\} \\
& =\widetilde{L}\left(a, b ; \Theta_{x, y}\right)\left\{\sum_{n=0}^{\infty} \sum_{k=n}^{\infty} \frac{(-1)^{k} q^{\left(\begin{array}{c}
k \\
2
\end{array}\right)} d^{k} t^{n}}{(q ; q)_{k}(q ; q)_{n-k}}(c ; q)_{k} p_{n-k}(v, u) p_{n}(y, x)\right\} \\
& =\widetilde{L}\left(a, b ; \Theta_{x, y}\right)\left\{\sum_{n=0}^{\infty} \sum_{k=0}^{\infty} \frac{(-1)^{k} q^{\left(\begin{array}{l}
k \\
2
\end{array}\right)} d^{k} t^{n+k}}{(q ; q)_{k}(q ; q)_{n}}(c ; q)_{k} p_{k}(v, u) p_{n+k}(y, x)\right\} \\
& =\widetilde{L}\left(a, b ; \Theta_{x, y}\right)\left\{\sum_{k=0}^{\infty} p_{k}(v, u) p_{k}(y, x) \frac{(-1)^{k} q^{\left(\begin{array}{l}
k \\
2
\end{array}\right)(c ; q)_{k}(d t)^{k}}}{(q ; q)_{k}} \sum_{n=0}^{\infty} p_{n}\left(y, q^{k} x\right) \frac{t^{n}}{(q ; q)_{n}}\right\} \\
& =\widetilde{L}\left(a, b ; \Theta_{x, y}\right)\left\{\sum_{k=0}^{\infty} p_{k}(v, u) p_{k}(y, x) \frac{(-1)^{k} q^{\left(\begin{array}{c}
k \\
2
\end{array}\right)(c ; q)_{k}(d t)^{k}}}{(q ; q)_{k}} \frac{\left(x t q^{k} ; q\right)_{\infty}}{(y t ; q)_{\infty}}\right\} \\
& =\widetilde{L}\left(a, b ; \Theta_{x, y}\right)\left\{\frac{(x t ; q)_{\infty}}{(y t ; q)_{\infty}} \sum_{k=0}^{\infty} \frac{(-1)^{k} q^{\left(\begin{array}{l}
k \\
2
\end{array}\right)(x / y, u / v, c ; q)_{k}}}{(x t ; q)_{k}} \frac{(d v y t)^{k}}{(q ; q)_{k}}\right\} \\
& \left.\left.=\widetilde{L}\left(a, b ; \Theta_{x, y}\right)\left\{\frac{(x t ; q)_{\infty}}{(y t ; q)_{\infty}}{ }_{3} \Phi_{3}\left[\begin{array}{c}
x / y, u / v, c ; \\
0,0, x t ;
\end{array}\right]\right\} ; v v y t\right]\right\}
\end{aligned}
$$

which evidently completes our demonstration of Theorem 3.5.

\section{CONCluding Remarks And ObSERVATions}

In our present investigation, we have constructed a pair of potentially useful homogeneous $q$-operators, namely, the homogeneous $q$-shift operator $\widetilde{E}\left(a, b ; D_{q}\right)$ and the homogeneous $q$-difference operator $\widetilde{L}\left(a, b ; \Theta_{x, y}\right)$. We then have successfully applied each of these homogeneous $q$-operators in order to represent and investigate a family of generalized Cauchy polynomials and a general form of the $q$-Hahn polynomials. In particular, we have derived several $q$-identities such as generating functions, extended generating functions, Mehler-type bilinear generating functions and Rogers-type formulas for the general families of $q$-polynomials which we have introduced in this paper. We have also considered relevant connections of the $q$-identities presented here with a number of known or new results associated with various specialized families of $q$-polynomials.

\section{REFERENCES}

[1] M.A. Abdlhusein, Two operator representations for the trivariate $q$-polynomials and Hahn polynomials, Ramanujan J. 40 (2016), 491-509.

[2] G.E. Andrews, The Theory of Partitions, Cambridge University Press, Cambridge, London and New York, 1985.

[3] S. Araci, M. Acikgöz, T. Diagana, H.M. Srivastava, A novel approach to obtaining new identities for the $\lambda$-extension of $q$-Euler polynomials arising from the $q$-umbral calculus, J. Nonlinear Sci. Appl. 10 (2017), 1316-1325. 
[4] J. Cao, H. M. Srivastava, Some $q$-generating functions of the Carlitz and Srivastava-Agarwal types associated with the generalized Hahn polynomials and the generalized Rogers-Szegö polynomials, Appl. Math. Comput. 219 (2013), 83988406.

[5] W.Y.-C. Chen, A.-M. Fu, B. Zhang, The homogeneous q-difference operator, Adv. Appl. Math. 31 (2003), 659-668.

[6] W.Y.-C. Chen, Z.-G. Liu, Parameter augmenting for basic hypergeometric series. II, J. Combin. Theory Ser. A 80 (1997), 175-195.

[7] G. Gasper, M. Rahman, Basic Hypergeometric Series, Second edition, Cambridge University Press, Cambridge, London and New York, 2004.

[8] J. Goldman, G.-C. Rota, On the foundations of combinatorial theory, IV: Finite vector spaces and Eulerian generating functions, Stud. Appl. Math. 49 (1970), 239-258.

[9] E.C. Ihrig, M.E.-H. Ismail, A q-umbral calculus, J. Math. Anal. Appl. 84 (1981), 178-207.

[10] W.P. Johnson, $q$-Extensions of identities of Abel-Rothe type, Discrete Math. 159 (1995), 161-177.

[11] R. Koekock, R. F. Swarttouw, The Askey-scheme of hypergeometric orthogonal polynomials and its $q$-analogue report (Preprint), Delft University of Technology, Delft, The Netherlands, 1998.

[12] S. Roman, The theory of the umbral calculus. I, J. Math. Anal. Appl. 87 (1982), 58-115.

[13] S. Roman, More on the umbral calculus, with emphasis on the $q$-umbral calculus, J. Math. Anal. Appl. 107 (1985), 222-254.

[14] H.L. Saad, A.A. Sukhi, Another homogeneous $q$-difference operator, Appl. Math. Comput. 215 (2010), $4332-4339$.

[15] H.L. Saad, A.A. Sukhi, The q-exponential operator, Appl. Math. Sci. 7 (2005), 6369-6380.

[16] L.J. Slater, Generalized Hypergeometric Functions, Cambridge University Press, Cambridge, London and New York, 1966.

[17] H.M. Srivastava, M.A. Abdlhusein, New forms of the Cauchy operator and some of their applications, Russian J. Math. Phys. 23 (2016), 124-134.

[18] H.M. Srivastava, P.W. Karlsson, Multiple Gaussian Hypergeometric Series, Halsted Press (Ellis Horwood Limited, Chichester); John Wiley and Sons, New York, Chichester, Brisbane and Toronto, 1985. 